Check for updates

Cite this: RSC Adv., 2019, 9, 34120

Received 4th September 2019 Accepted 19th September 2019

DOI: $10.1039 / c 9 r a 07089 c$

rsc.li/rsc-advances

\title{
Near-field infrared microscopy of nanometer-sized nickel clusters inside single-walled carbon nanotubes $\uparrow$
}

\author{
Gergely Németh, ${ }^{\star a}$ Dániel Datz, ${ }^{a}$ Áron Pekker, (D) ${ }^{a}$ Takeshi Saito, (D) ${ }^{b}$ \\ Oleg Domanov, (D) ${ }^{c}$ Hidetsugu Shiozawa, (D) ${ }^{c d}$ Sándor Lenk, (DD e Béla Pécz, ${ }^{f}$ \\ Pál Koppa ${ }^{e}$ and Katalin Kamarás (D) ${ }^{a}$
}

\begin{abstract}
Nickel nanoclusters grown inside single-walled carbon nanotubes (SWCNT) were studied by infrared scattering-type scanning near-field optical microscopy (s-SNOM). The metal clusters give high local contrast enhancement in near-field phase maps caused by the excitation of free charge carriers. The experimental results are supported by calculations using the finite dipole model, approximating the clusters with elliptical nanoparticles. Compared to magnetic force microscopy, s-SNOM appears much more sensitive to detect metal clusters inside carbon nanotubes. We estimate that these clusters contain fewer than $\approx 700 \mathrm{Ni}$ atoms.
\end{abstract}

One of the unique applications of carbon nanotubes is their use as nanocontainers for various encapsulated species. Nanoscale metal clusters present a special perspective in this regard as the tubes give both a natural constraint and an effective protection from the environment. Following the early description of the filling procedure, ${ }^{1}$ a considerable variety of both single- and multiwalled nanotubes combined with several metals were produced (for a review, see ref. 2), and their structural, ${ }^{3}$ magnetic $^{4}$ and superconducting ${ }^{5,6}$ properties investigated. Metallocenes in single-walled carbon nanotubes (SWCNTs) ${ }^{7}$ represent a special class among these hybrid systems, as they constitute nanoreactors for both metal cluster formation ${ }^{8}$ and inner nanotube growth. ${ }^{9}$ In a recent study ${ }^{10}$ upon annealing nickelocene encapsulated in SWCNTs, superparamagnetic nickel clusters were formed that are considered as high performance single domain magnets with high coercivity. Here we measure such clusters by scattering-type near-field optical microscopy (s-SNOM) to both probe the metallicity of such

anstitute for Solid State Physics and Optics, Wigner Research Centre for Physics, Hungarian Academy of Sciences, Konkoly-Thege M. u. 29-33, H-1121 Budapest, Hungary. E-mail: nemeth.gergely@wigner.mta.hu

${ }^{b}$ Nanomaterials Research Institute, AIST, 1-1-1 Higashi, Tsukuba 305-8565, Japan 'Faculty of Physics, University of Vienna, Boltzmanngasse 5, A-1090 Vienna, Austria ${ }^{d}$ J. Heyrovsky Institute of Physical Chemistry, Academy of Sciences of the Czech Republic, Dolejskova 3, CZ-182 23 Prague 8, Czech Republic

${ }^{e}$ Department of Atomic Physics, Budapest University of Technology and Economics, Budafoki út 8, H-1111 Budapest, Hungary

Institute for Technical Physics and Materials Science, Centre for Energy Research, Hungarian Academy of Sciences, Konkoly-Thege M. u. 29-33, H-1121 Budapest, Hungary

$\dagger$ Electronic supplementary information (ESI) available. See DOI: $10.1039 / \mathrm{c} 9 \mathrm{ra} 07089 \mathrm{c}$ small nanostructures and to establish the sensitivity of the method.

Details of encapsulation of $\mathrm{Ni}(\mathrm{II})$ acetylacetonate in e-Dips single walled carbon nanotubes with tube diameters of $1.7 \pm$ $0.1 \mathrm{~nm}$ are given in ref. 10. The encapsulated molecules were transformed to nickel clusters by annealing. The size of these clusters can be controlled by the annealing temperature: in order to get fewer but well separated, long clusters we heated the sample in vacuum at $700{ }^{\circ} \mathrm{C}$ for 2 hours. Previous results using similar conditions ${ }^{10}$ showed the formation of nickel clusters with aspect ratio ranging approximately from 1 to 15 . Near-field microscopy was performed on samples deposited on a silicon substrate by vacuum filtration. ${ }^{11}$

In order to follow and control the annealing process we verified the disappearance of $\mathrm{Ni}(\mathrm{II})$ acetylacetonate molecules using attenuated total reflection (ATR) spectroscopy in the midinfrared (MIR) region (Fig. S1†). The disappearance of the acetylacetonate-related peaks indicates the successful decomposition of the molecules and the possible formation of $\mathrm{Ni}$ clusters.

We also observed nickel clusters being created inside the nanotubes via transmission electron microscopy (TEM). The nanotubes were dispersed in toluene, sonicated for 1 hour and collected onto a TEM grid with ultrathin carbon film, then images were taken using both a JEOL 3010 and an FEI THEMIS microscope. A typical image is shown in Fig. S2. $\dagger$ The clusters look spatially separated enough to enable the measurement of the optical response via s-SNOM where the possible resolution limit is around $20 \mathrm{~nm}$.

We applied scattering-type near-field optical microscopy (sSNOM) to image nano-sized nickel atom clusters inside single 
walled carbon nanotubes based on their infrared optical response with spatial resolution well beyond the diffraction limit. The s-SNOM setup (Neaspec $\mathrm{GmbH}$ ) is based on an atomic force microscope with a metal-coated tip illuminated from the side by a focused laser beam (in our case, the source was an infrared $\left(980 \mathrm{~cm}^{-1}\right)$ quantum cascade laser (QCL)). The illuminated metal tip acts as an optical antenna ${ }^{\mathbf{1 2}}$ and enhances the electric field under the tip as depicted in Fig. 1 . The extension of this well-localized, high amplitude electric field depends on the tip apex radius. ${ }^{13}$ As this nano-sized light probe is scanned in the proximity to the surface, an optical interaction occurs between the probe and the sample.

This interaction results in propagating waves via scattering from the volume below the probing tip. The amplitude and the phase of the scattered wave are determined by the local optical properties of the sample. ${ }^{\mathbf{1 4 , 1 5}}$ The very weak near-field scattered light is then demodulated at the higher harmonics of the tip oscillation frequency and further analyzed using pseudoheterodyne detection ${ }^{\mathbf{1 6}}$ based on a Michelson-type interferometer, shown in Fig. S3.†

This complex setup enables the simultaneous measurement of the sample topography and both the amplitude and the phase of the near-field scattered light. The s-SNOM microscopy yields very high wavelength-independent spatial resolution $(\approx 20 \mathrm{~nm})$ and high optical response that gives the opportunity to study nanostructures consisting of only a few hundred atoms.

Magnetic force microscopy (MFM) was also performed with a separate AFM instrument (Bruker Dimension Icon) using a standard Bruker MESP magnetic AFM tip.

In the applied mid-infrared spectral region the near-field signal originates from the excitation of free charge carriers. In common metals, such excitations result in a frequencyindependent high phase contrast compared to the silicon substrate. Although carbon nanotubes show near-field contrast themselves, ${ }^{17,18}$ their conductivity is negligible compared to real metals like nickel, therefore we do not expect observable contribution from the nanotube walls.

In order to predict the near-field contrast we performed calculations based on the extended finite dipole model (EFDM). ${ }^{19,20}$ In the infrared region where the wavelength is much longer than the characteristic size of the tip-particlesubstrate system, the scattering problem can be approximated as an electrostatic problem at each time step (Rayleigh

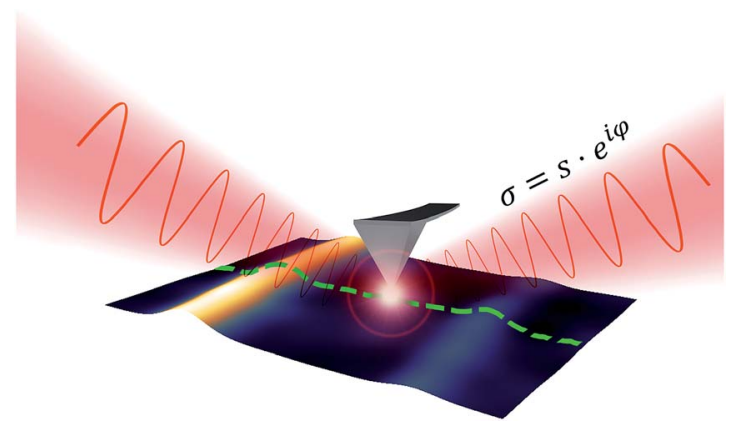

Fig. 1 Illustration of the near-field scattering process and the illuminated probing tip. scattering). The system is modeled as depicted in Fig. 2. The tip is approximated as a prolate spheroid, the nanotube as an infinite long cylinder. The nickel clusters are also cylinderlike objects as previous studies ${ }^{\mathbf{1 0}}$ suggested. In order to fit them to the analytical model we replaced them with prolate spheroids because their polarizability is very close to that of a cylinder.

Neglecting the effect of the carbon nanotube (see above), the main part of the near-field interaction occurs between the probing tip, the nickel cluster and the substrate. The electric field is considered to be perpendicular to the surface of the substrate as the tip enhances the electric field component that is parallel to its axis of revolution. In such a model we can take into account the electric field response of the nickel cluster with several dipoles generated inside the nickel nanoparticle by the tip and the mirror charges of the substrate. Those dipoles are described by the local electric field and the polarizability of the object ( $p=\alpha E_{\text {loc }}$ ). The polarizability of prolate spheroids, perpendicular to their semi-major axis is given by the formula: ${ }^{21}$

$$
\alpha_{\perp}=\frac{4}{3} R^{2} L\left(\frac{2 R}{L}\right)^{2} \frac{\varepsilon-1}{1+N_{\perp}(\varepsilon-1)}
$$

Here $\varepsilon$ is the dielectric permittivity of the nanoparticle at the wavelength of interest, $R$ is the radius, and $L$ is the length of the elliptical nanoparticle, and $N_{\perp}$ is the depolarization factor. The latter describes how much the internal field within the spheroid is attenuated by the polarization and it depends on the geometry of the object. This can be expressed as

$$
N_{\perp}=\frac{1}{2}\left(1-N_{\|}\right)
$$

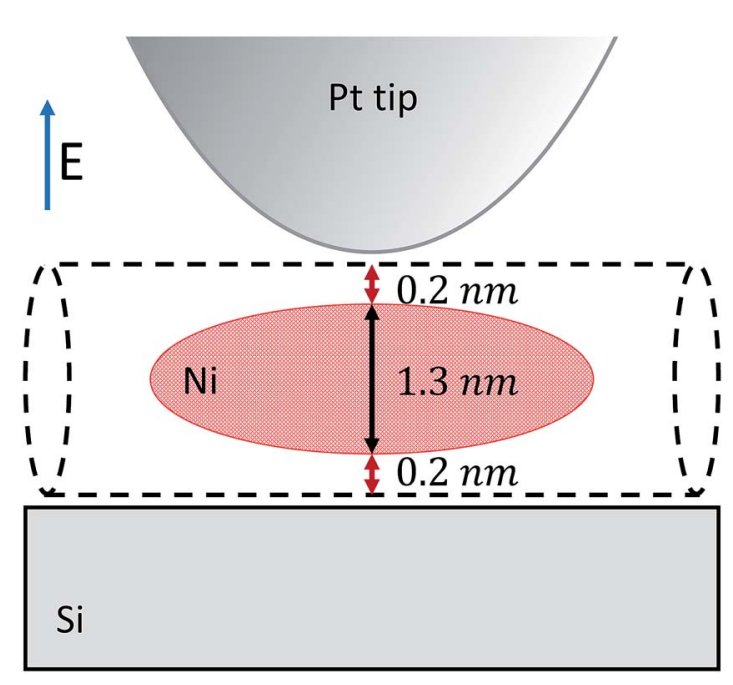

Fig. 2 Schematic representation of the probe-sample configuration used in our analytical model. The nickel cluster is modeled as a prolate spheroid. The nanotube wall (including the van der Waals distances) separates the tip and the substrate from the side of the nickel cluster. The average diameter of the e-Dips nanotubes is $1.7 \mathrm{~nm}$ thus the nickel cluster is considered to be $1.3 \mathrm{~nm}$ in diameter. 


$$
N_{\|}=\frac{1-e^{2}}{1 e^{3}}\left(\ln \frac{1+e}{1-e}-2 e\right)
$$

where $e=\sqrt{1-\left(\frac{R}{L / 2}\right)}$ is the eccentricity of the spheroid. The diameter is chosen to be $D=1.3 \mathrm{~nm}$ to fit inside a nanotube with diameter $1.7 \mathrm{~nm}$. The dielectric permittivity of nickel was determined from the Drude model with a size-dependent term for the damping constant: ${ }^{22}$

$$
\varepsilon(\omega, R)=1-\frac{\omega_{\mathrm{p}}{ }^{2}}{\omega^{2}+\mathrm{i} \omega \gamma+\mathrm{i} \omega C \frac{v_{\mathrm{F}}}{R}}
$$

where $v_{\mathrm{F}}$ is the Fermi velocity and $C$ is a factor that depends on the electron scattering process inside the particle. The Drude model parameters for nickel were taken from ref. 22.

These parameters were used in the EFDM model to calculate the 3rd harmonic demodulated near-field phase contrast of nickel nanoparticles compared to the silicon substrate versus the aspect ratio of the nanoparticle $(L / D)$. The result is presented in Fig. 3.

Fig. 3 illustrates that the near-field phase contrast does not vary noticeably with the aspect ratio: the near-field contrast changes only by $\Delta \varphi_{\mathrm{O} 3}=0.001$ rad until it starts to saturate when the aspect ratio is around four. This amount of change cannot be detected under the present experimental conditions.

Results on as-prepared Ni(II) acetylacetonate-filled nanotubes transferred onto silicon are shown in Fig. 4. We were looking for nanotube bundles with as small diameter as possible. We expect no phase signal from such bundles as Ni(II) acetylacetonate molecular vibrations are too weak to provide an observable near-field signal. We found that nanotube bundles smaller than $10 \mathrm{~nm}$ have no contrast on the near-field optical maps. The figure presents the AFM topography and the O3 nearfield phase map of the sample with multiple carbon nanotube bundles. The lack of a near-field signal verifies our predictions.

Next, we applied the annealing process $\left(700^{\circ} \mathrm{C}, 2 \mathrm{~h}\right)$ to this sample to create nickel clusters ${ }^{10}$ and repeated the optical characterization of the nanotubes. During the process the surface morphology of the sample changed substantially and it

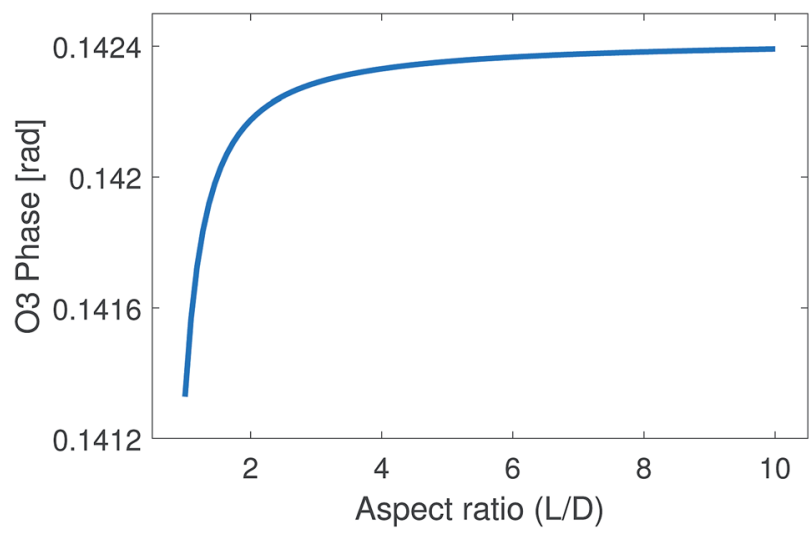

Fig. 3 Third harmonic (O3) near-field phase signal of a nickel nanoparticle with different aspect ratios $(L / D)$ for fixed $D=1.3 \mathrm{~nm}$, calculated from the EFDM model. All data were normalized to the signal from the silicon substrate.

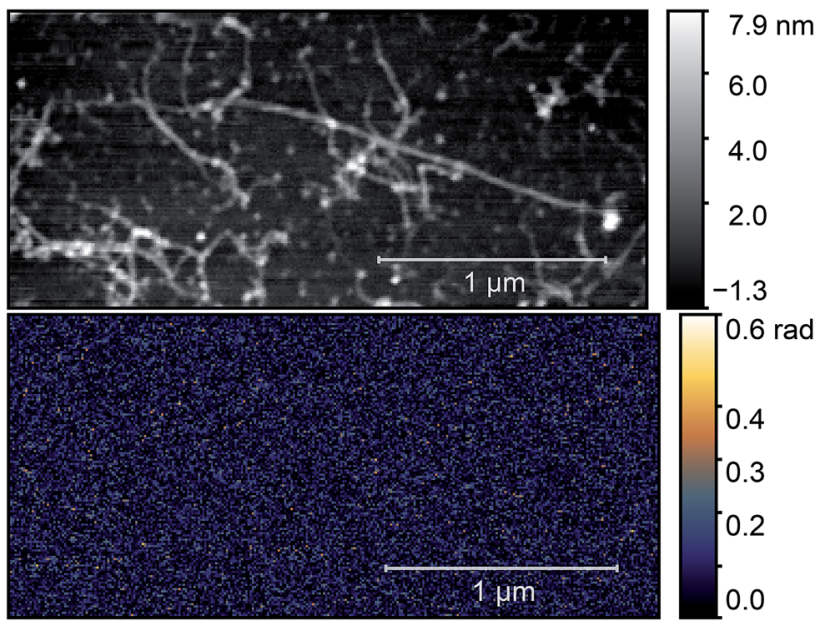

Fig. 4 AFM topography (top) and $\mathrm{O} 3$ near-field map (bottom) of a typical $\mathrm{Ni}\left({ }^{\prime \prime}\right)$ acetylacetonate-filled carbon nanotube bundle before the annealing process. Optical images were taken with a $\nu=980 \mathrm{~cm}^{-1}$ illuminating laser.

was not possible to find the same nanotube. Therefore, we searched for nanotubes bundles with identical diameter in the two images. We chose the bundle in the middle of Fig. 4 with a height of $3 \mathrm{~nm}$ as standard. Since the diameter of an individual e-Dips nanotube is $1.7 \mathrm{~nm}$, such a bundle would consist of three nanotubes. In Fig. 5, we show the AFM topography and the third harmonic phase signal of a bundle of similar size.

The figure shows a typical nanotube bundle in the middle with diameter of $3 \mathrm{~nm}$. Since the diameter of an individual eDips nanotube is $d \approx 1.7 \mathrm{~nm}$, bundles with $d=3 \mathrm{~nm}$ probably consist of three nanotubes.

As the optical image demonstrates, bright, high contrast spots alternate along the nanotubes, with regions where no optical signal is found. These high contrast spots correspond to the transmission electron microscopy images which showed inhomogeneous spatial distribution of nickel clusters along the nanotube bundles. Our measurements show remarkable agreement with the analytical model. Phase contrast values of the brightest spots are $\varphi_{\mathrm{O} 3}=0.139 \pm 0.01 \mathrm{rad}$, to be compared with a calculated value of $\varphi \approx 0.142 \mathrm{rad}$. Other examples of phase maps can be seen in Fig. 6, where the location of the nickel clusters can be easily identified. We also found very few nanotubes with no contrast, indicating that the filling of most tubes was sufficient to obtain nickel clusters. The contrast values vary within the sample; smaller values possibly correspond to smaller diameter clusters that do not fill the nanotube perfectly, larger contrast, in turn, could indicate multiple clusters measured together at the bottom of the tip. We also managed to do sequential mapping at different wavenumbers. From these images we extracted the average phase value of the clusters in a representative nanotube at every measured wavenumber. Fig. $\mathrm{S} 4 \dagger$ shows a near-field phase spectrum acquired this way, together with that calculated from the EFDM. The high, frequency-independent phase value through the measured spectral range indicates metallic behavior. 

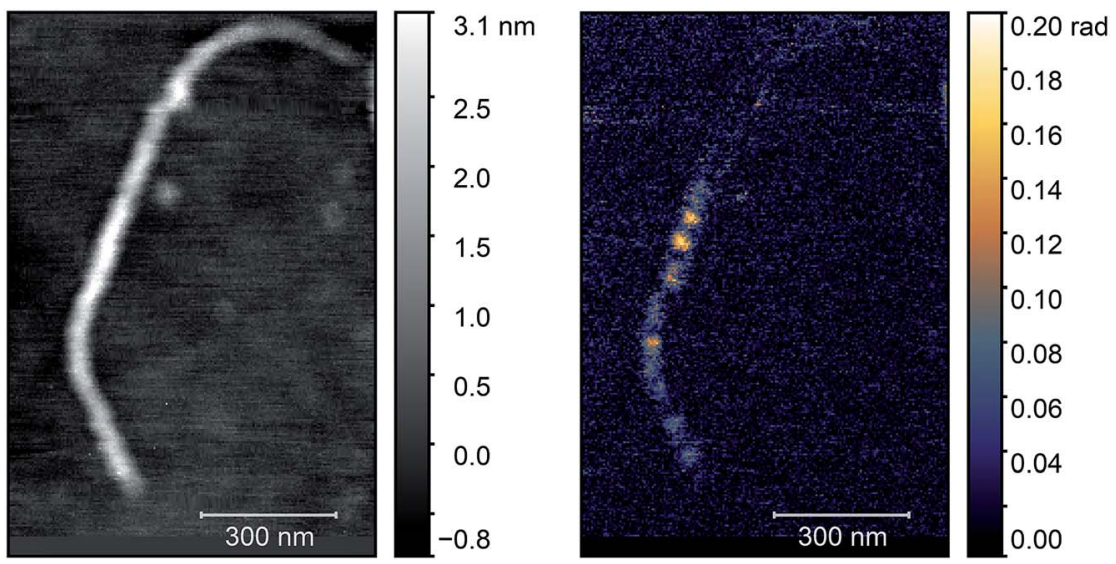

Fig. 5 AFM topography (left) and O3 near-field map (right) of a typical carbon nanotube bundle after the annealing process. Optical images were taken with a $\nu=980 \mathrm{~cm}^{-1}$ illuminating laser.

We also tried to locate the nickel clusters by magnetic force microscopy (MFM). This technique was already applied to investigate nanoparticles with magnetic behavior. ${ }^{23-25}$ As the excellent magnetic properties of these nickel clusters were already demonstrated, ${ }^{10}$ we expected to obtain the signal of nickel clusters in the MFM phase images as magnetic dipoles give bright and dark spots at their opposite poles. Fig. 7 displays the AFM topography, the MFM and s-SNOM measurements on the same nanotube bundle. We repeated the MFM measurements with different tip lift height $(15,25,35,50,70,130 \mathrm{~nm})$ until the topographic related phase appeared. We did not find any sign of nickel clusters with MFM probing, indicating the higher sensitivity of the optical method.

If we assume that the aspect ratio of a nickel cluster is four (this was the saturation limit in the calculated near-field phase) and we treat it like a cylinder shaped cluster of face centered cubic (f.c.c.) structured nickel, we can roughly estimate the number of atoms measured in one spot. We used the lattice constant of f.c.c. nickel, $0.35 \mathrm{~nm}$ (ref. 26) and calculated how many cubes can fit in the above mentioned cylinder. This
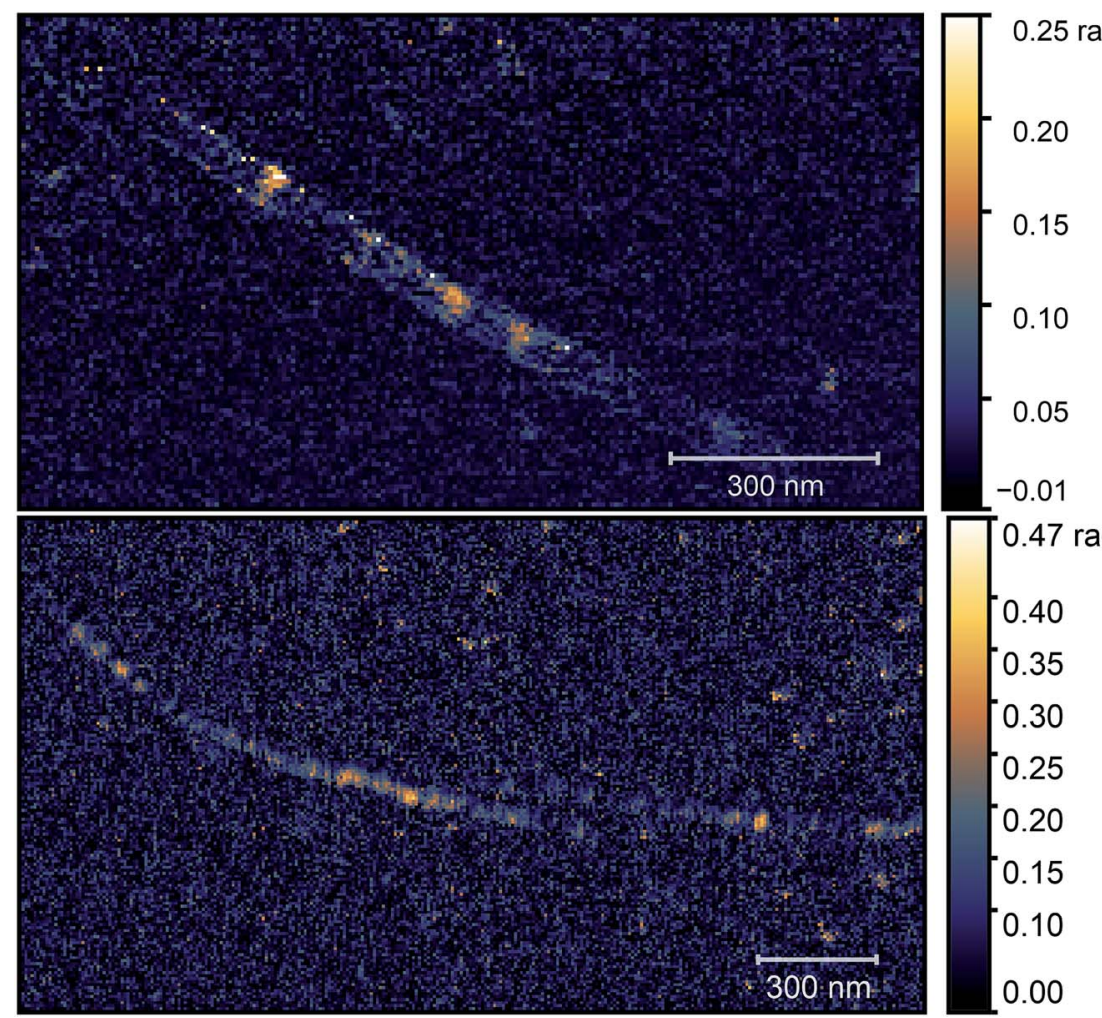

Fig. 6 O3 optical phase maps of two different carbon nanotube bundles. Images were taken with a $\nu=980 \mathrm{~cm}^{-1}$ illuminating laser. The presence of nickel clusters is very obvious as they cause high phase contrast. 


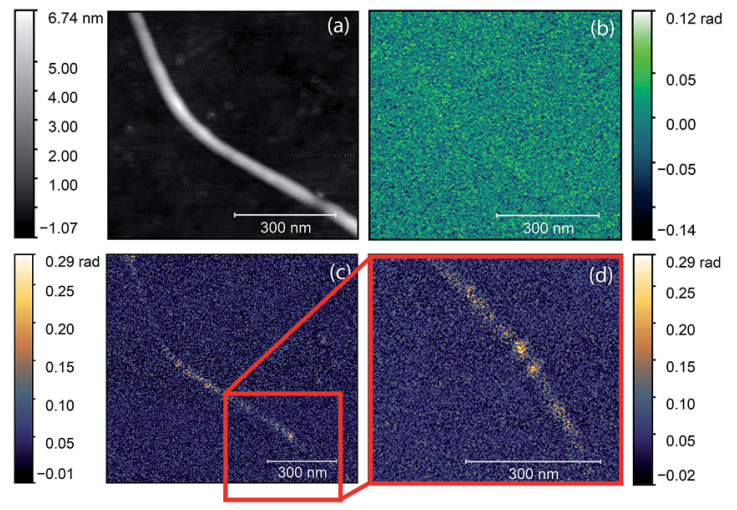

Fig. 7 AFM topography (a), MFM phase (b), O3 near-field phase (c) of the same nanotube bundle at the same place. (d) presents the highlighted area of (c). The figures clearly show contrast in optical images while magnetic microscopy is not sensitive to nickel clusters of this size.

number was then multiplied by four because the unit cell of an f.c.c. crystal contains 4 atoms, giving the number of atoms to be around 644. We find that near-field optical probing gives reliable information about the location of nickel clusters inside carbon nanotubes even in the case of such a small amount of material.

In summary, we observed nickel nanoclusters grown inside single walled carbon nanotubes via near-field microscopy based on their infrared optical properties. We found that these measurements are very sensitive to the presence of the metallic phase. With a tip-defined spatial resolution of $25 \mathrm{~nm}$, we were able to detect optical signals from objects of a few $\mathrm{nm}$ in size, containing less than 700 atoms. Our modified EFDM model gives phase contrasts close to the measured values and is found to reliably predict the optical signal of nanoparticles. We also detected nickel clusters in most of the nanotubes, consistent with electron microscopy results which indicate the good filling ratio of the nanotubes.

\section{Conflicts of interest}

There are no conflicts to declare.

\section{Acknowledgements}

Research financed by the Hungarian National Research Fund (OTKA) No. SNN 118012. Transmission electron microscopy was supported by the VEKOP-2.3.3-15-2016-00002 project. H. S. acknowledges funding from the Austrian Science Fund (FWF) P30431-N36. Á. P. gratefully acknowledges support from the János Bolyai Fellowship of the Hungarian Academy of Sciences and from the National Research, Development and Innovation Office - NKFIH PD 121320 and NKFIH FK-125063.

\section{Notes and references}

1 J. Sloan, J. Hammer, M. Zwiefka-Sibley and M. Green, Chem. Commun., 1998, 1998, 347-348.
2 C. Soldano, Prog. Mater. Sci., 2015, 69, 183-212.

3 L. Guan, K. Suenaga, S. Okubo, T. Okazaki and S. Iijima, J. Am. Chem. Soc., 2008, 130, 2162-2163.

4 M. Lutz, U. Weissker, F. Wolny, C. Müller, M. Löffler, T. Mühl, A. Leonhardt, B. Büchner and R. Klingeler, J. Phys.: Conf. Ser., 2010, 200, 072062.

5 L. Jankovič, D. Gournis, P. N. Trikalitis, I. Arfaoui, T. Cren, P. Rudolf, M. Sage, T. T. M. Palstra, B. Kooi, J. De Hosson, M. A. Karakassides, K. Dimos, A. Moukarika and T. Bakas, Nano Lett., 2006, 6, 1131-1135.

6 N. Tombros, L. Buit, I. Arfaoui, T. Tsoufis, D. Gournis, P. N. Trikalitis, S. J. van der Molen, P. Rudolf and B. J. van Wees, Nano Lett., 2008, 8, 3060-3064.

7 L.-J. Li, A. N. Khlobystov, J. G. Wiltshire, G. A. D. Briggs and R. J. Nicholas, Nat. Mater., 2005, 4, 481-485.

8 Y. F. Li, R. Hatakeyama, T. Kaneko, T. Izumida, T. Okada and T. Kato, Nanotechnology, 2006, 17, 4143-4147.

9 H. Shiozawa, T. Pichler, A. Grüneis, R. Pfeiffer, H. Kuzmany, Z. Liu, K. Suenaga and H. Kataura, Adv. Mater., 2008, 20, 1443-1449.

10 H. Shiozawa, A. Briones-Leon, O. Domanov, G. Zechner, Y. Sato, K. Suenaga, T. Saito, M. Eisterer, E. Weschke, W. Lang, H. Peterlik and T. Pichler, Sci. Rep., 2015, 5, 15033.

11 Z. Wu, Z. Chen, X. Du, J. M. Logan, J. Sippel, M. Nikolou, K. Kamaras, J. R. Reynolds, D. B. Tanner, A. F. Hebard and A. G. Rinzler, Science, 2004, 305, 1273-1276.

12 P. Bharadwaj, B. Deutsch and L. Novotny, Adv. Opt. Photonics, 2009, 1, 438-483.

13 R. Hillenbrand and F. Keilmann, Appl. Phys. Lett., 2002, 80, 25-27.

14 R. Hillenbrand and F. Keilmann, Phys. Rev. Lett., 2000, 85, 3029-3032.

15 J. M. Stiegler, Y. Abate, A. Cvitkovic, Y. E. Romanyuk, A. J. Huber, S. R. Leone and R. Hillenbrand, ACS Nano, 2011, 5(8), 6494-6499.

16 R. Hillenbrand and F. Keilmann, Appl. Phys. Lett., 2006, 89, 101124.

17 G. Németh, D. Datz, H. M. Tóháti, Á. Pekker and K. Kamarás, Phys. Status Solidi B, 2016, 253, 2413-2416.

18 G. Németh, Á. Pekker, D. Datz, H. Tóháti, K. Otsuka, T. Inoue, S. Maruyama and K. Kamarás, Phys. Status Solidi $B, 2017,254,1700433$.

19 N. Ocelic, Ph.D. thesis, Technical University Munich, 2007. 20 A. Cvitkovic, Ph.D. thesis, Technische Universität München, 2009.

21 J. Venermo and A. Sihvola, J. Electrost., 2005, 63, 101-117.

22 D. M. Arboleda, J. M. J. Santillan, L. J. M. Herrera, D. Muraca, D. C. Schinca and L. B. Scaffardi, J. Phys. D: Appl. Phys., 2016, 49, 075302.

23 J. Garcia, A. Thiaville and J. Miltat, J. Magn. Magn. Mater., 2002, 249, 163-169.

24 J. M. Kinsella and A. Ivanisevic, Langmuir, 2007, 23, 38863890.

25 T. Wang, Y. Wang, Y. Fu, T. Hasegawa, F. S. Li, H. Saito and S. Ishio, Nanotechnology, 2009, 20, 105707.

26 W. P. Davey, Phys. Rev., 1925, 25, 753-761. 